\title{
Visual outcome and complications after cataract extraction in Saudi Arabia
}

Department of Ophthalmology, College of Medicine, King Saud University, and King Khaled Eye Specialist Hospital, Riyadh, Saudi Arabia

M F Al Faran

Correspondence to: Mubarak F Al Faran, MD, King Khaled Eye Specialist Hospital, PO Box 7191 , Riyadh 11462 , Saudi Arabia.

Accepted for publication 11 September 1989

\author{
Mubarak F Al Faran
}

\begin{abstract}
Cataract is the leading cause of blindness in Saudi Arabia. The author studied 1383 patients who had undergone cataract extraction (total of 1520 procedures) from October 1985 to March 1986 at the King Khaled Eye Specialist Hospital, Riyadh, Saudi Arabia. All patients were observed for at least two months. Of 1520 eyes $555(37 \%)$ achieved a visual acuity of $20 / 40$ or better, $309(20 \%)$ a visual acuity of 20/50-20/60 after surgery, and $656(43 \%)$ eyes a visual acuity of $20 / 70$ or less. The number of eyes that had intraoperative complications was $176(12 \%)$. The number of eyes that had postoperative complications was 539 (35\%). Some eyes had more than one complication. The main factors responsible for failure to achieve visual acuity of $20 / 40$ or better were: (1) error of refraction in $492(32 \%)$ eyes; (2) preexisting corneal scarring and opacity in 211 (14\%) eyes; (3) climatic droplet keratopathy in 134 (9\%); and (4) presumed visual loss due to glaucoma in $134(9 \%)$ eyes.
\end{abstract}

Cataracts are an enormous medical, financial, and social burden. Annually at least 300000 to 400000 new, visually disabling cataracts occur in the United States, and 5 to 10 million occur worldwide. ${ }^{1}$ Throughout the world cataracts are most prevalent in areas where the amounts of annual and daily sunlight are high. ${ }^{23}$ In Saudi Arabia cataract has been found to be the leading cause of blindness, accounting for $55 \%$ of all cases. Furthermore, it is the second most important cause of visual loss, accounting for $35 \cdot 5 \%{ }^{4}$ The visual outcome and complication rates are the objective means of assessing the success of cataract surgery. ${ }^{56}$ While cataract surgery is considered to be safe and effective, irreversible blindness either from the complications of advanced, untreated cataract, or from the complications of surgery, may occur. ${ }^{1}$ In addition associated ocular diseases increase the risk of complications and reduce the chance of a good visual outcome. ${ }^{7}$

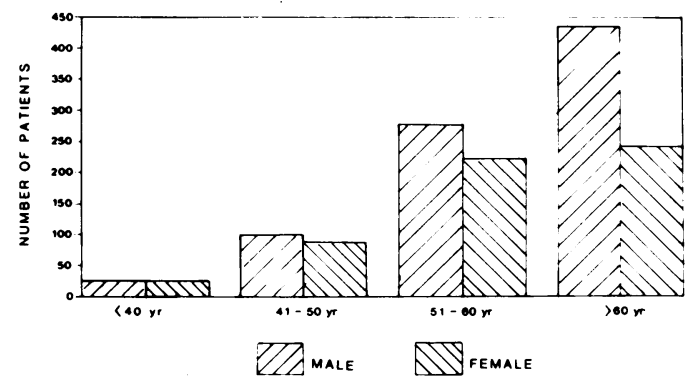

Figure 1: Age and sex distribution of 1383 patients who underwent cataract extraction.
It has been noted that the number of cataract surgeries performed at the King Khaled Eye Specialist Hospital (KKESH) is large. It also has been thought that the visual outcome differed from that reported in western countries. It was therefore decided to study patients undergoing cataract extraction at KKESH. The major objectives of this study are: (1) to determine the visual outcome and the factors influencing visual outcome after cataract extraction and (2) to assess the complications of cataract extraction.

\section{Patients and methods}

A total of 1520 consecutive (senile or presenile) cataract extractions from 1383 patients were analysed from October 1985 to March 1986 at the King Khaled Eye Specialist Hospital. This retrospective case review included patients aged 35 years or older.

CLINICAL EXAMINATION AND FOLLOW-UP A diagnosis of cataract was made after full ophthalmic examination, including biomicroscopy, tonometry, and indirect ophthalmoscopy. Ultrasonography was performed to assess the posterior segment of the eye if the media were opaque and for intraocular lens power calculation. After cataract surgery the patients were observed daily for two to three days in the hospital. After discharge from the hospital they were observed weekly for two weeks and then monthly for at least two months. Patients excluded from this study were those who gave a history of ocular trauma, were less than the age of 35 years, or who showed retinal detachment by ultrasonography. In addition patients who did not complete a follow-up for at least two months were excluded.

\section{SURGICAL PROCEDURES}

All surgical procedures were performed by standard microsurgical techniques for cataract surgery, with or without intraocular lens implantation. There were 1469 (95\%) extracapsular cataract extractions (ECCE) and 76 $(5 \%)$ intracapsular cataract extractions (ICCE). A total of $144(10 \%)$ eyes had other ocular procedures performed at the time of cataract extraction. Forty-three patients (3\%) had trabeculectomy and 93 patients $(6 \%)$ had anterior vitrectomy, because of the vitreous loss. Other procedures such as keratectomy for climatic droplet keratopathy and pterygium excision were performed in eight patients. The procedures were performed by several surgeons with various levels of experience. 
Results

Of the 1383 patients in this study $831(60 \%)$ were male and $552(40 \%)$ were female. The male to female ratio was $1 \cdot 5: 1$ (Fig 1 ). Their ages ranged from 35 to 95 years, mean 64 years. The followup ranged from two months to 38 months, mean nine months, after date of surgery. The number of patients aged 40 or more years was 1337 (97\%).

Table I shows the visual acuities before cataract surgery and the visual acuities achieved at least two months after cataract extraction in 1520 eyes. Of these, 555 (37\%) eyes attained $20 / 40$ or better and $309(20 \%)$ eyes attained a visual acuity of $20 / 50-20 / 60 ; 455(30 \%)$ eyes attained $20 / 70$ $20 / 200$. Fifty (3\%) eyes had a poor visual acuity of between 20/300 and 20/400, and $151(10 \%)$ eyes had a visual acuity of between hand motion and light perception (HM-LP).

Seven hundred and fifteen eyes (47\%) had complications. Some eyes had more than one complication.

Intraoperative complications. 176 eyes $(11 \cdot 6 \%)$ had intraoperative complications, which included vitreous loss in $97(6.4 \%)$; rupture of the posterior capsule in $85(5.6 \%)$; zonular dialysis in $23(1.5 \%)$; significant bleeding in 22 (1.4\%); Descemet's membrane detachment in 18 (1.2\%); and other complications in $23(1 \cdot 5 \%)$ eyes (Table II).

Postoperative complications. 539 eyes (35.5\%) had postoperative complications. These included posterior capsule opacity in $150(9.9 \%)$ eyes; peaking pupil in $130(8.6 \%)$; high intraocular pressure in $90(5.9 \%)$; corneal oedema in $68(4.5 \%)$; ruptured or loose suture in $47(3.2 \%)$; and other complications in $62(4.0 \%)$ eyes (Table III).

A total of 965 eyes (62\%) did not achieve a visual acuity of $20 / 40$ or better. Table IV shows the factors responsible for failure to achieve a visual acuity of $20 / 40$ or better. It was found that $492(32 \%)$ eyes did not have a final refraction. 211 eyes (14\%) had corneal scarring and opacity; $162(11 \%)$ had climatic droplet keratopathy;

TABLE I Visual acuity before and after cataract extraction

\begin{tabular}{lccccc}
\hline & \multicolumn{2}{c}{ Preop. VA } & & \multicolumn{2}{l}{ Postop. VA } \\
\cline { 2 - 3 } \cline { 5 - 6 } Visual acuity & $\begin{array}{l}\text { Number of } \\
\text { eyes }\end{array}$ & $(\%)$ & & $\begin{array}{l}\text { Number of } \\
\text { eyes }\end{array}$ & $(\%)$ \\
\hline $20 / 15-20 / 40$ & 0 & $(0)$ & & 555 & $(37)$ \\
$20 / 50-20 / 60$ & 12 & $(1)$ & 309 & $(20)$ \\
$20 / 70-20 / 200$ & 232 & $(15)$ & & 455 & $(30)$ \\
$20 / 300-20 / 400$ & 118 & $(8)$ & 50 & $(3)$ \\
CF-LP & 1158 & $(76)$ & 151 & $(10)$ \\
Total & 1520 & $(100 \%)$ & 1520 & $(100 \%)$ \\
\hline
\end{tabular}

$\mathrm{VA}=$ visual acuity. $\mathrm{CF}=$ counting fingers. $\mathrm{LP}=$ light perception.

TABLE II Intraoperative complications (176 eyes) *

\begin{tabular}{lll}
\hline Complications & Number & $\%$ (of 1520) \\
\hline Rupture of capsule & 85 & 5.6 \\
Vitreous loss & 97 & 6.4 \\
Zonule dialysis & 23 & 1.5 \\
Detachment of Descemet's membrane & 18 & 1.2 \\
Bleeding & 22 & 1.4 \\
Others: & 23 & 1.5 \\
Iris complication & 15 & 1.0 \\
AC collapse & 2 & $0 \cdot 1$ \\
Global perforation & 1 & $0 \cdot 1$ \\
Others & 5 & 0.3 \\
\hline
\end{tabular}

*291 complications occurred in 176 eyes. $\mathrm{AC}=$ anterior chamber.
TABLE III Postoperative complications (539 eyes) ${ }^{\star}$

\begin{tabular}{lcl}
\hline Complications & Number & $\%$ (of 1520) \\
\hline Wound leak & 12 & $0 \cdot 8$ \\
Iris prolapse & 9 & $0 \cdot 6$ \\
Peaking pupil & 130 & $8 \cdot 6$ \\
External infection & 16 & $1 \cdot 0$ \\
High IOP & 90 & $5 \cdot 9$ \\
Hyphaema & 32 & $2 \cdot 1$ \\
Retinal detachment & 7 & $0 \cdot 5$ \\
Ruptured/loose suture & 47 & $3 \cdot 2$ \\
Astigmatism & 36 & $2 \cdot 4$ \\
IOL displacement & 21 & $1 \cdot 4$ \\
Postcapsule opacity & 150 & $9 \cdot 9$ \\
Residual cortex & 50 & $3 \cdot 2$ \\
Corneal oedema & 68 & $4 \cdot 5$ \\
Others & 62 & $4 \cdot 0$ \\
\hline
\end{tabular}

$\star 730$ complications occurred in 539 eyes.

TABLE IV Factors adversely affecting visual acuity (>20/40 after cataract extraction)

\begin{tabular}{lcc}
\hline Complications & $\begin{array}{l}\text { Number } \\
\text { of eyes }\end{array}$ & $\begin{array}{c}\%(\text { of } \\
1520)\end{array}$ \\
\hline No final refraction & 492 & 32.4 \\
Corneal scar/opacity & 211 & 13.9 \\
Climatic droplet keratopathy & 162 & $10 \cdot 6$ \\
Glaucoma/cupped disc & 134 & $8 \cdot 8$ \\
Diabetic retinopathy & 61 & $4 \cdot 0$ \\
Post staphyloma/myopic retinal degeneration & 67 & $4 \cdot 4$ \\
Senile macular degeneration & 44 & 2.9 \\
Macular oedema & 21 & 1.4 \\
Retinal detachment & 14 & 0.5 \\
Optic atrophy & 16 & 1.0 \\
Amblyopia & 14 & 0.9 \\
Vitreous haemorrhage & 12 & 0.8 \\
Vitreous opacities & 11 & 0.7 \\
Others & 89 & 5.8 \\
\hline
\end{tabular}

134 (9\%) had glaucoma with cupping of the disc, probably responsible for the level of visual acuity; 67 (4\%) had posterior staphyloma and myopic retinal degeneration; $61(4 \%)$ had diabetic retinopathy; and 51 (3\%) had posterior capsular opacity. Non-myopic macular degeneration was found in $44(3 \%)$ eyes, macular oedema in $21(1 \%)$, retinal detachment in $14(1 \%)$, optic atrophy in $16(1 \%)$, amblyopia in $14(1 \%)$, vitreous haemorrhage in $12(1 \%)$, vitreous opacity in $11(1 \%)$, and a few other rare factors.

\section{Discussion}

Eye disease, visual impairment, and blindness have long been recognised as major causes of human suffering and disability in the Kingdom of Saudi Arabia. The National Eye Survey conducted by the King Khaled Eye Specialist Hospital in 1984 revealed that $1.5 \%$ of the population in Saudi Arabia is blind by World Health Organisation (WHO) standards (less than 3/60 vision in the better eye with best available correction). ${ }^{4}$ At the national level the leading causes of blindness are cataract, trachoma, refractive error, corneal scars, iatrogenic from medical or surgical treatment, and glaucoma. ${ }^{4}$ The visual outcome after cataract extraction depends on several factors: corneal transparency, the status of the vitreous, and the functional integrity of both the retina and optic nerve.

The visual outcome in selected series of patients after senile cataract surgery is usually better than in unselected series of patients. Bernth-Petersen ${ }^{6}$ reported visual acuity of $6 / 12$ $(20 / 40)$ or better in $81.6 \%$ of his selected series of patients with senile cataract after surgery. This is due to the avoidance of patients with pre-existing 
ocular diseases such as corneal opacity, optic atrophy, or retinal detachment. In our study it is interesting to note that 555 eyes $(36 \%)$ achieved a visual acuity of $20 / 40$ or better after cataract surgery, and only 864 eyes (56\%) achieved a useful vision of $20 / 60$ or better. This was due to many reasons, the first being that final refraction was not performed on 492 eyes (32\%).

This points to a problem that may face ophthalmologists working in developing countries. Certainly it highlights a common clinical problem in Saudi Arabia, which is supported by the finding of the National Eye Survey, that the error of refraction was the first most common cause of visual loss in Saudi Arabia. ${ }^{4}$ This dilemma might be attributed to the customs and cultural habits of the people in Saudi Arabia, where the majority are not accustomed to wearing glasses. Sometimes ophthalmologists do not determine their patients' refractive errors, nor do they prescribe corrective spectacles or lenses, believing that their patients will not use them.

The second reason is the corneal scarring and opacities from past inflammations and infections. These include trachoma, smallpox, and measles. In spite of the significant reduction of the prevalence of trachoma and the eradication of smallpox, these corneal opacities are the result of diseases acquired at an earlier time in the Kingdom's history, when medical care was not of today's high standards.

The third reason is climatic droplet keratopathy $(\mathrm{CDK})$, which was found in $162(11 \%)$ eyes. The presence of climatic droplet keratopathy, or spheroidal degeneration, involving the central cornea contributed significantly to the reduction of vision. Climatic droplet keratopathy is commoner in males than in females. In Saudi Arabia presumably males are more exposed to ultraviolet radiation than are females, who are usually protected by wearing veils, or by being outdoors less often than males.

It is interesting to note that the male to female ratio in our study is $1 \cdot 5: 1$. This is contrary to the fact that cataract in our general population was found to be more common among females than in males. ${ }^{4}$ However, this discrepancy may be explained by the fact that this is a hospital based study, and in this country males seek medical advice more than females, who do not drive and who generally stay at home.

Noteworthy is the high prevalence of cataract among patients under the age of 60 years. This might be explained by excessive exposure to sunlight. Ultraviolet light has been considered as a predisposing factor for cataract formation. This was confirmed by Collman and his coworkers. ${ }^{8}$ They found an increased risk factor of cataract formation among dark eyed subjects, which is consistent with reports of high prevalence rates of cataract in areas populated with dark eyed persons who are exposed to sunlight.

Senile macular degeneration (SMD) is one of the main factors in the reduction of visual acuity after cataract surgery in western countries. This does not seem to be the case in developing countries. The prevalence of senile macular degeneration in the cataract population was $13 \cdot 2 \%$ in one study. ${ }^{6}$ Age related macular degeneration in our study was found only in 44 eyes $(2 \cdot 8 \%)$. This may mean a lower prevalence of senile macular degeneration than in America and Europe in the same age group of patients.

It is important to note that the majority (76\%) of the eyes had a preoperative visual acuity of from counting fingers to light perception. These eyes had mature senile cataract and required prompt surgical intervention to prevent hypermature cataract and its unpleasant sequelae. Lack of visualisation of the posterior segment coupled with a poor history does not allow us to select patients free of preoperative ocular complications which later contribute to reduced visual acuity after cataract surgery. With the high number of cataract patients being treated, we expect to see patients with immature cataract, and we shall be able to identify many preoperative factors responsible for reduced visual acuity.

Correction of refractive error is a major challenge, and all efforts should be made to encourage people to use glasses. It is also important to recommend a routine use of the intraocular lens after cataract surgery whenever possible. As cataract is not only the leading cause of blindness in Saudi Arabia but also the second most important cause of overall visual loss, special care during and after operation is expected to decrease the visual loss due to cataract surgery. This special care is of paramount importance in a country where in the past an iatrogenic element was a considerable factor in blindness. ${ }^{4}$

Finally, this study points to a major clinical problem which needs an early solution to decrease the rate of blindness in Saudi Arabia. The backlog of blinding cataract cases should be given priority for surgery. It is expected that, once the cataract backlog is controlled, glaucoma may become the main cause of blindness. Public health education should be developed so that patients with visual loss know how to seek help. The eye departments at different hospitals in the Kingdom of Saudi Arabia should be upgraded and provided with proper facilities and enough qualified physicians appointed to perform effective and safe cataract surgery.

In conclusion, the proper evaluation of patients for cataract surgery and the complete visual rehabilitation of those patients are important in both the immediate and the longterm postoperative period. Failure of a cataract operation is not only a personal tragedy but also a major socioeconomic burden to the individual and to his society.

1 Sommer A. Cataracts as an epidemiologic problem. Am $\mathcal{f}$ Ophthalmol 1977; 83: 334-9.

2 Taylor HR. The environment and the lens. Br $\mathcal{F}$ Ophthalmol $1980 ; 64: 303-10$

3 Hiller R, Giacommeti L, Yuen K. Sunlight and cataract: An epidemiologic investigation. Am $\mathcal{f}$ Epidemiol 1977; 105: 450-9.

4 Tabbara KF, Ross-Degnan D. Blindness in Saudi Arabia. FAMA 1986; 255: 3378-84.

5 Vernon S, Cheng $\mathrm{H}$. Comparison between the complications of cataract surgery following local anaesthesia with short stay and general anaesthesia with five days hospitalisation. $\mathrm{Br} \mathcal{F}$ Ophthalmol 1985; 69: 360-3.

6 Bernth-Petersen P. Outcome of cataract surgery: I. A prospective/observational study. Acta Ophthalmol (Kbh) 1982; 60: 235-42.

7 Meredith T, Maumenee E. Review of 1000 cases of intracapsular cataract extractions: I. Complications. Ophthalmic Surg 1979; 10: 32-41.

8 Collman GW, Shore DL, Shy CM, Checkoway H, Luria AS. Sunlight and other risk factors for cataracts: an epidemiologic study. Am $\mathcal{F}$ Public Health 1988; 78: 1459-62. 